\title{
Qualidade de vida dos idosos inseridos em uma universidade aberta à terceira idade
}

\author{
Life quality of old-aged people inserted at an elderly opened university
}
Calidad de vida de las personas mayores insertadas en una universidad abierta para
personas mayores

Mariana Barbosa da Silva ${ }^{1 *}$, Rafael Rocha de Azeredo ${ }^{1}$, Emilenny Lessa dos Santos ${ }^{1}$, Cyndi Myrelle da Silva Barros Romão ${ }^{1}$, Maria Karolina de Souza Rodrigues ${ }^{2}$, Maria Mayara Stephanne de Medeiros Freitas ${ }^{1}$, Francyele Alves da Paixão Nobre ${ }^{3}$.

\section{RESUMO}

Objetivo: Avaliar a qualidade de vida dos idosos inseridos em uma Universidade Aberta à Terceira Idade. Métodos: Trata-se de um estudo de campo, analítico, de corte transversal, com abordagem quantitativa. A amostra foi composta por 132 idosos participantes de uma Universidade Aberta à Terceira Idade no Estado de Alagoas, escolhidos por amostragem probabilística aleatória simples. Foram utilizadas duas escalas validadas sobre a capacidade funcional e qualidade de vida: WHOQOL-bref e WHOQOL-old. A análise dos dados foi feita a partir do programa Microsolft Excel. Resultados: Observou-se que 84\% dos participantes são do sexo feminino e $73,5 \%$ possui entre 60 e 69 anos. A maior média obtida no instrumento WHOQOL-bref foi o domínio "psicológico" com 3,94, o domínio "meio ambiente" obteve a menor média com 3,44. No módulo WHOQOL-old a faceta com maior escore foi "habilidades sensoriais" com $76 \%$ e menor escore foram "autonomia" e "morte e morrer" com $65 \%$ cada. Conclusão: Apesar de serem considerados idosos ativos, o resultado não foi satisfatório em diversas facetas, evidenciando a importância de mais ações que favoreçam a promoção da saúde e qualidade de vida dos idosos.

Palavras-chave: Envelhecimento populacional, Qualidade de vida, Universidade.

\section{ABSTRACT}

Objective: To evaluate the quality of life of old-aged people inserted at an Elderly Opened University. Methods: It's about an analithycal field research, cross sectional, with quantitative approach. The sample was composed by 132 old-aged people from an Elderly Opened University at the State of Alagoas, chosen by simple random probabilistic sample. Two validated scales about functional capacity and life quality were used: WHOQOL-bref and WHOQOLold. The data analysis was made from the Microsoft Excel program. Results: It was observed that $84 \%$ of the participants are female and $73.5 \%$ are between 60 and 69 years old. The highest average obtained in the WHOQOLbref instrument was the "psychological" domain with 3.94, the "environment" domain obtained the lowest average with 3.44. In the WHOQOL-old module the facet with the highest score was "sensory abilities" with $76 \%$ and the lowest score were "autonomy" and "death and dying", both with 65\% each. Conclusion: Although the elderly are considered active, the result wasn't satisfactory in many facets, evidencing the importance of more action that favors the health promotion and quality of life of the old-aged people.

Keywords: Demographic aging, Quality of life, Universities.

\section{RESUMEN}

Objetivo: Evaluar la calidad de vida de los adultos mayores insertados en una Universidad Abierta a la Tercera Edad. Métodos: Se trata de un estudio de campo, analítico, transversal, con enfoque cuantitativo. La muestra estuvo conformada por 132 ancianos participantes de una Universidad Abierta para Ancianos del Estado de Alagoas, elegidos por muestreo probabilístico aleatorio simple. Se utilizaron dos escalas validadas de capacidad funcional y calidad de vida: WHOQOL-bref y WHOQOL-old. El análisis de los datos se realizó mediante el programa Microsolft Excel. Resultados: Se observó que el 84\% de los participantes son mujeres y el 73,5\% tienen entre 60 y 69 años. El promedio más alto obtenido en el instrumento WHOQOL-bref fue el dominio "psicológico" con 3.94, el dominio "ambiente" obtuvo el promedio más bajo con 3.44. En el módulo WHOQOL-old, la faceta con mayor

\footnotetext{
${ }^{1}$ Universidade Estadual de Ciências da Saúde de Alagoas (UNCISAL), Maceió - AL.

${ }^{*}$ E-mail: barbosamari@hotmail.com

${ }^{2}$ Centro Universitário Cesmac (CESMAC), Maceió - AL.

${ }^{3}$ Centro Universitário Tiradentes (UNIT), Maceió - AL.
}

SUBMETIDO EM: 9/2020 
puntuación fue "habilidades sensoriales" con $76 \%$ y la menor puntuación fue "autonomía" y "muerte y morir" ambas con $65 \%$ cada una Conclusión: A pesar de ser considerado anciano activo, el resultado no fue satisfactorio en varias facetas, mostrando la importancia de más acciones que favorezcan la promoción de la salud y la calidad de vida de las personas mayores.

Palabras clave: Envejecimiento poblacional, Calidad de vida, Universidad.

\section{INTRODUÇÃO}

O envelhecimento populacional no Brasil está associado a importantes alterações demográficas, sociais e econômicas que acarretam à formulação e implementação de políticas públicas no âmbito da proteção social ao idoso, segundo Sousa MS (2015). A população brasileira ganhou 4,8 milhões de idosos desde 2012, superando a marca de 30,2 milhões em 2017. Em 2012, a população com 60 anos ou mais era de 25,4 milhões. Os 4,8 milhões de novos idosos em cinco anos correspondem a um crescimento de $18 \%$ desse grupo etário, que tem se tornado cada vez mais representativo no Brasil.

A profunda alteração nos padrões demográficos apresenta grande visibilidade na esfera pública, alertando para problemas em vários domínios, desde os sistemas de proteção e de segurança social, prestação de cuidados da saúde, rede de equipamentos e de serviços até as políticas de apoio à família. Afinal, o envelhecimento populacional caracteriza desafios à sustentabilidade dos sistemas públicos de proteção social (MIRANDA, GMD, et al., 2016).

Com o processo de envelhecimento, que é descrito como as mudanças morfofuncionais que surgem ao longo da vida, ocorrem alterações bioquímicas, histológicas, citológicas, fisiológicas e imunológicas; que levam os indivíduos idosos a serem portadores de doenças crônico-degenerativas, prejudicando sua qualidade de vida. Apesar de o processo de envelhecimento não estar necessariamente relacionado a doenças e incapacidades (LINDER JUNIOR E TRINDADE JLA, 2013).

As doenças crônicas não transmissíveis (DCNT) se constituem como o grupo de doenças de maior magnitude no país, atingindo, especialmente, as populações mais vulneráveis, segundo Brasil (2011). Portanto, a criação de estratégias que possibilitem uma vida mais saudável, deve ser prioridade na atenção à saúde dos idosos, visando não só minimizar o impacto da doença na vida do indivíduo, mas proporcionar mais qualidade na vida dos idosos (TIER, GC et al., 2014).

A qualidade de vida (QV) é definida como o discernimento da pessoa sobre sua configuração na vida, nos meios da cultura ou no sistema de valores onde estão inseridos e em relação aos seus objetivos, perspectivas, padrões e responsabilidades (WHOQOL GROUP, 1995). Tendo em vista que as doenças e as incapacidades funcionais geram impacto sobre a família, o sistema de saúde e o cotidiano dos idosos, deve-se buscar tardar sua evolução com 0 intuito de garantir longevidade com autonomia, independência e maior qualidade (TAVARES DMS, DIAS FA, 2012).

Dentro desta perspectiva, a inserção de medidas para ajudar os idosos a se manterem ativos, saudáveis e inseridos socialmente torna-se uma necessidade e, sobretudo, uma responsabilidade por parte das universidades, pelo seu papel de formação educacional, política, de integração e de agente social (ROQUE, FP, et al., 2011).

No início da década de 1970, na Universidade de Ciências Sociais de Toulouse, França, foi criada a primeira Universidade da Terceira Idade (The University Of The Third Age). A partir daí, o movimento das Universidades Abertas da Terceira Idade (UnTAl), se difundiu por todo o mundo, alcançando milhões de pessoas idosas que participam de várias atividades intelecto-culturais que contribuem para um envelhecimento ativo e, consequentemente, para uma velhice bem sucedida (ROQUE, FP, et al., 2011).

No Brasil, no começo da década de 1980 foram seguidas as primeiras ações pelo modelo francês das UnATI, com expressivo crescimento em todo o território nacional na década de 1990. No ano de 2006 foi implementada em uma Universidade do Estado de Alagoas, com a oferta de oficinas de caráter manual, físico e cognitivo, com o intuito de promover a integração universidade-idoso-comunidade, envolvendo vários segmentos da comunidade universitária e da comunidade em geral (ROQUE FP, et al., 2011). 
Considerando o constante crescimento da população idosa, bem como o crescimento das morbidades nesse grupo etário, faz-se imprescindível que os profissionais da saúde tenham dados sobre a qualidade de vida da população idosa, para que ações sejam traçadas visando melhorar a terapêutica empregada, assim como, fundamentalmente uma atuação voltada para a prevenção e controle dessas doenças, bem como, a identificação precoce dos fatores ambientais de risco que possam influenciar na vida dos idosos, visando, a construção de um envelhecimento saudável.

Dado o texto supracitado, este estudo tem a seguinte questão de pesquisa: como se apresenta a qualidade de vida dos idosos inseridos na Universidade Aberta à Terceira Idade do estado de Alagoas? $O$ objetivo foi avaliar a qualidade de vida dos idosos participantes da Universidade Aberta à Terceira Idade do estado de Alagoas.

\section{MÉTODOS}

Trata-se de um estudo de campo, analítico, de corte transversal, com abordagem quantitativa. A amostra total é de 132 entrevistados, escolhidos por amostragem probabilística aleatória simples. A pesquisa foi realizada no Programa de Extensão Universidade Aberta à Terceira Idade- UNCISATI da Universidade Estadual de Ciências da Saúde de Alagoas- UNCISAL. Esta pesquisa foi submetida à apreciação e aprovada pelo Comitê de Ética em Pesquisa da Universidade Estadual de Ciências da Saúde de Alagoas, sob o número do parecer: $1.614 .543 / 2016$.

Inicialmente, para a condução da coleta foi aplicado o Termo de Consentimento Livre e Esclarecido (TCLE) em duas vias e foram utilizados dois questionários: o instrumento The World Health Organization quality of life assessment (WHOQOL)-bref e o módulo WHOQOL-old, escalas utilizadas internacionalmente para avaliação da QV. No WHOQOL-bref consta 26 questões, sendo duas questões gerais de qualidade vida e as 24 restantes representam cada uma das 24 facetas que compõem o instrumento original (WHOQOL-100). O WHOQOL-bref é composto por quatro domínios: físico, psicológico, relações sociais e meio ambiente.

As facetas: capacidade de trabalho, atividades da vida cotidiana, sono e repouso, mobilidade, energia e fadiga, dependência de medicamentos ou tratamento, dor e desconforto, compõem o domínio físico; o domínio psicológico é composto pelas facetas: sentimentos negativos, autoestima, imagem corporal e aparência, pensar, aprender, memória e concentração, espiritualidade/religião/crenças pessoais e sentimentos positivos; o domínio relações sociais com as facetas: relações pessoais, atividade sexual e suporte (apoio) social; já o domínio meio ambiente é composto por: transporte, cuidados de saúde e sociais: disponibilidade e qualidade, ambiente no lar, participação em, e oportunidades de recreação/lazer, oportunidades de adquirir novas informações e habilidades, recursos financeiros, ambiente físico: (poluição/ruído/trânsito/clima) e segurança física e proteção.

O WHOQOL-bref não gera um escore total, pois o grupo de pesquisa WHOQOL acredita que a variância entre os domínios indica qual delas mais influenciou a QV. Quanto maior o escore, melhor a percepção de QV. Neste instrumento o resultado aparece somente em média (1 a 5). Para calcular o domínio foram somados os valores das facetas e divididos de acordo com a quantidade de facetas em cada domínio. Classificação: necessita melhorar (quando for 1 até 2,9); regular (3 até 3,9); boa (4 até 4,9) e muito boa (5) (WHOQOL, 1995).

O WHOQOL-old consiste em 24 itens da escala Likert, sendo composto por seis facetas: funcionamento sensório, autonomia, atividades passadas, presentes e futuras, participação social, morte e morrer e intimidade. Para cada faceta, o escore pode oscilar de 4 a 20 pontos; somado aos 24 itens, ele gera um escore bruto.

Portanto, os escores dessas seis facetas ou os valores dos 24 itens podem ser combinados para produzir um escore total (global) para a QV em idosos. Com isso, quanto maior o escore, maior a qualidade de vida, quanto menor o escore, significará menor qualidade de vida.

Existem três formas de apresentar os dados: uma é em forma de total (de 4 a 20); outra é a média (1 a 5); outra é percentual ( 0 a 100). Para os resultados em média: necessita melhorar (quando for 1 até 2,9); regular (3 até 3,9 ); boa (4 até 4,9) e muito boa (5). Resultados em \% de 0 a 100, quanto maior a porcentagem (mais 
perto de $100 \%$ ) melhor a qualidade de vida. Os valores foram calculados a partir do resultado da análise em média e porcentagem.

Durante a realização da coleta, os sujeitos da pesquisa foram abordados antes ou após as oficinas ofertadas pelo projeto de extensão. Quando o respondente não entendeu o significado de alguma pergunta o entrevistador releu a pergunta de forma lenta, não sendo utilizados sinônimos ou "explicações em outras palavras da questão" (aplicação assistida). Quando o respondente não apresentou condições de ler o questionário em função de suas condições de saúde ou de alfabetização, o questionário foi lido pelo entrevistador. As informações coletadas são absolutamente confidenciais e anônimas, uma vez que foram seguidos todos os critérios que constam na Resolução 466/12 do CNS/MS.

Como critério de inclusão nesta pesquisa foram delimitadas as pessoas que tinham 60 anos ou mais, de ambos os sexos e que estivessem matriculados na Universidade Aberta à Terceira Idade. Foram excluídos da pesquisa os alunos afastados do programa de extensão. As variáveis consideradas foram as seguintes: autonomia, físico, psicológico, relações sociais, meio ambiente, funcionamento sensório, intimidade, atividades passadas, presentes e futuras, morte e morrer e participação social. Após a coleta, os dados foram descritos de forma absoluta, discutidos, analisados, agrupados e tratados estatisticamente através do programa Microsolft Excel 2010 que permite criar questionários, entrar dados, analisar dados, criar mapas, tabelas e gráficos de maneira simples e conveniente com várias variáveis e menor chance de erro.

\section{RESULTADOS}

Entre os 132 sujeitos participantes deste estudo, houve a predominância de $84 \%$ do sexo feminino em relação aos sujeitos do sexo masculino que teve $16 \%$. Em relação a faixa etária, observou-se que a participação dos idosos entre 60 e 69 anos foi de 73,5\%, os que têm entre 70 e 79 anos apresentou uma porcentagem de $23,5 \%$ e com menor índice os idosos entre 80 e 89 anos com apenas $3 \%$ do total de sujeitos entrevistados. Na avaliação da QV, realizada através do instrumento WHOQOL-bref, a média de satisfação com a qualidade de vida foi de 4,15 , já a satisfação com a saúde obteve uma média de 3,77 . Na Figura 1 observa-se as médias por domínios do instrumento WHOQOL-bref. O domínio com escore mais alto foi o psicológico, com uma média de 3,94. O domínio com menor escore foi o meio ambiente com uma média de 3,44 .

Figura 1 - Valores em média obtidos por domínio através do instrumento WHOQOL-bref dos idosos inseridos na Universidade Aberta à Terceira Idade, $n=132$. 2016-2017.

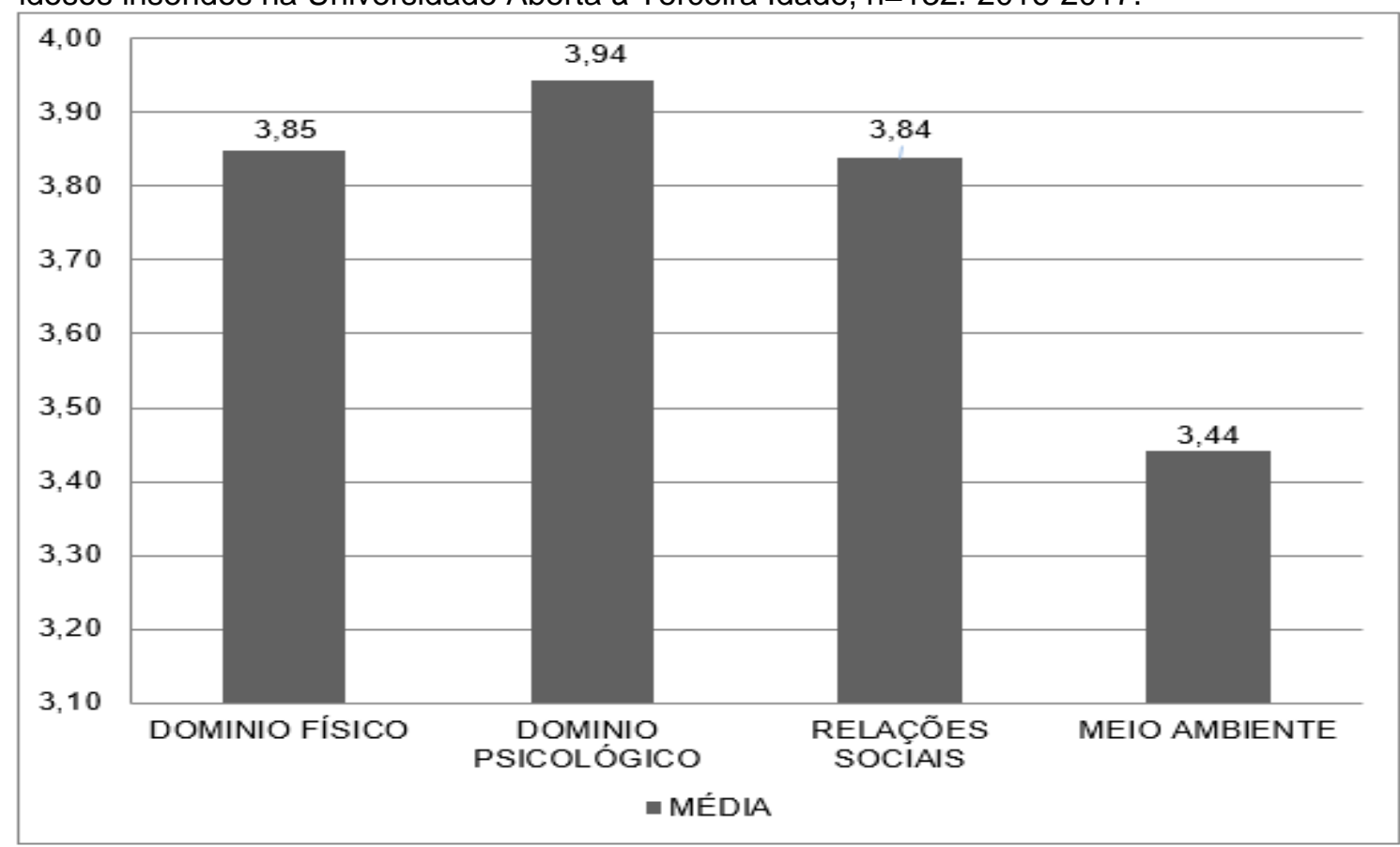

Fonte: Silva MB, et al., 2020. 
A Figura 2 apresenta as facetas do domínio físico do instrumento WHOQOL-bref. Nota-se que os maiores escores foram mobilidade, com uma média de 4,17, atividades da vida cotidiana, com uma média de 4,06, sono e repouso com 3,98 e capacidade de trabalho com 3,94. Enquanto as facetas com os menores escores foram dependência de medicamentos ou tratamento com 3,29, energia e fadiga com 3,61 e dor e desconforto com uma média de 3,87 .

Figura 2 - Valores obtidos nas facetas do domínio físico através do instrumento WHOQOL-bref dos idosos inseridos na Universidade Aberta à Terceira Idade, $n=132$. 2016-2017.

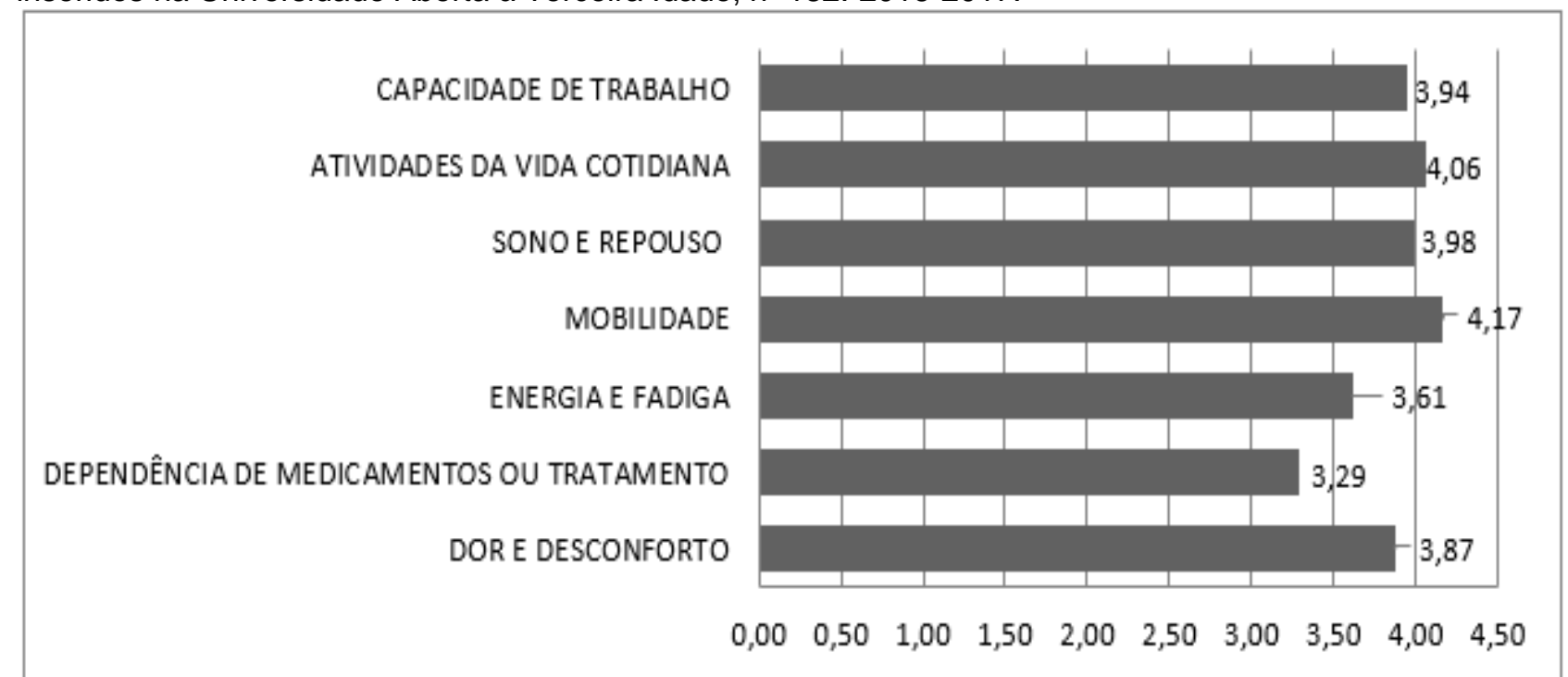

Fonte: Silva MB, et al., 2020.

Observa-se na Figura 3 as facetas do domínio psicológico do instrumento WHOQOL-bref. Os maiores escores foram autoestima, com uma média de 4,28, espiritualidade/religião/crenças pessoais com 4,18 e imagem corporal e aparência, com uma média de 4,16 . As facetas que apresentaram menores escores foram pensar, aprender, memória e concentração com 3,49, sentimentos positivos com 3,61 e sentimentos negativos com uma média de 3,95.

Figura 3 - Valores obtidos nas facetas do domínio psicológico através do instrumento WHOQOL-bref dos idosos inseridos na Universidade Aberta à Terceira Idade, n=132. 2016-2017.

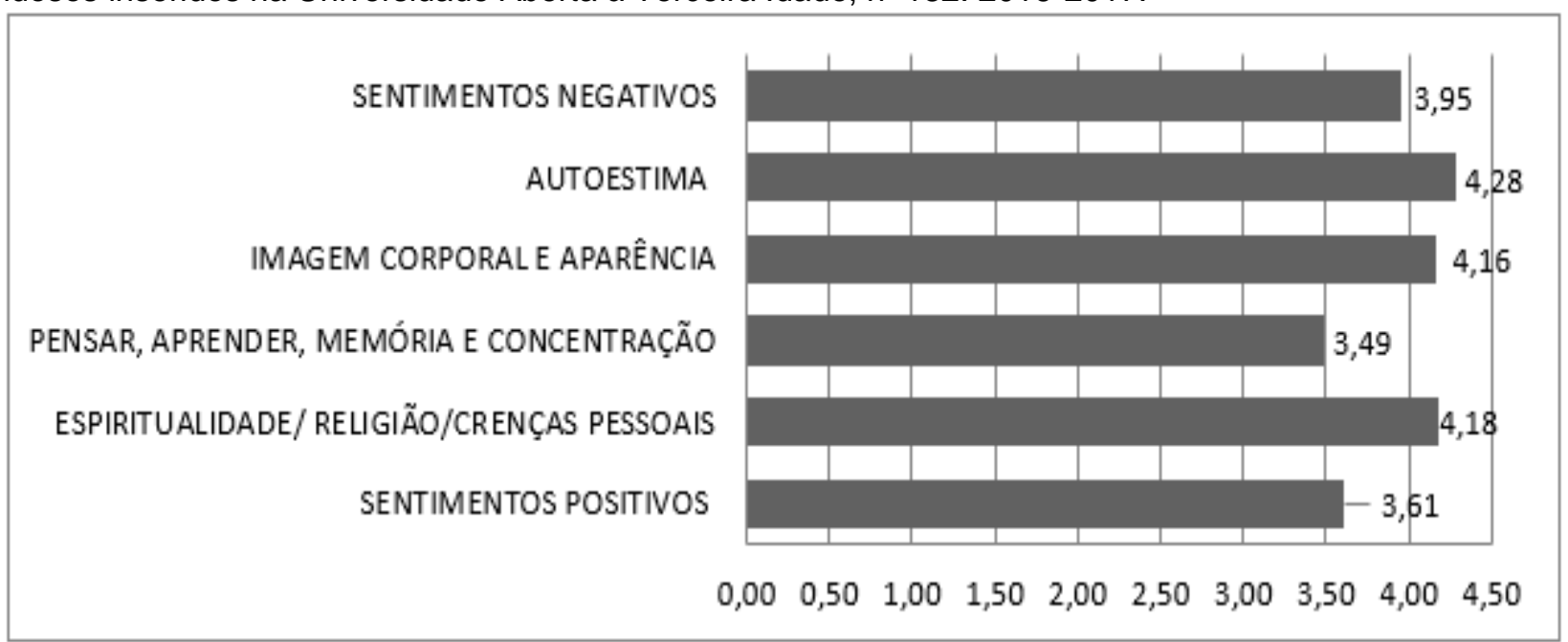

Fonte: Silva MB, et al., 2020.

As facetas do domínio relações sociais do instrumento WHOQOL-bref (Figura 4) mostra as relações pessoais com uma média de 4,19, suporte (apoio) social com 4,03, já atividade sexual apresenta-se com 3,29. 
Figura 4 - Valores obtidos nas facetas do domínio relações sociais através do instrumento WHOQOL-bref dos idosos inseridos na Universidade Aberta à Terceira Idade, $\mathrm{n}=132$. 2016-2017.

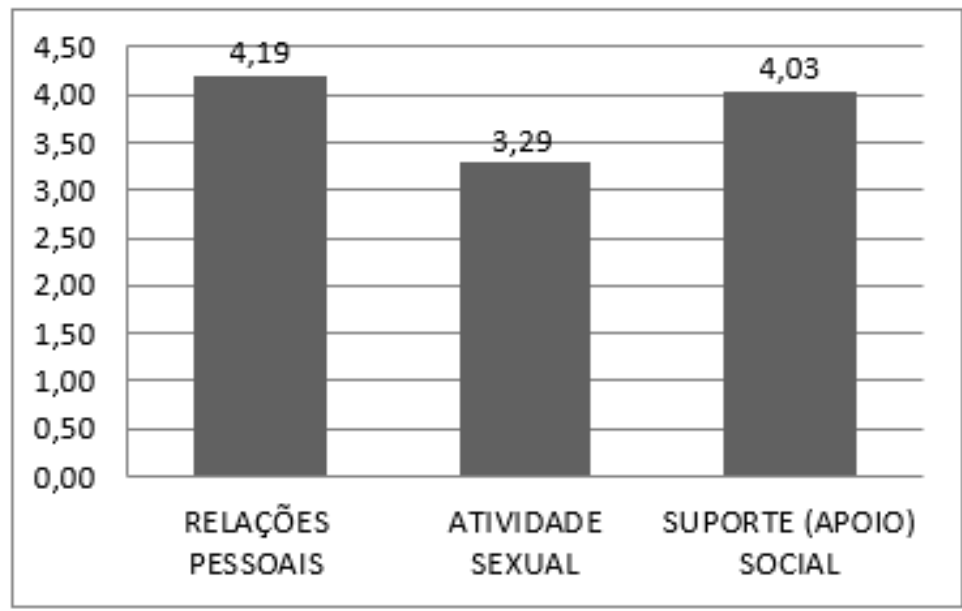

Fonte: Silva MB, et al., 2020.

Observa-se na Figura 5 as facetas do domínio meio ambiente do instrumento WHOQOL-bref. Apenas uma faceta, ambiente no lar, obteve uma média satisfatória de 4,06, as demais se apresentaram abaixo: segurança física e proteção com 3,66, oportunidades de adquirir novas informações e habilidades com uma média de 3,53, ambiente físico: poluição/ruído/trânsito/clima com 3,48, participação em, e oportunidades de recreação/lazer 3,30, recursos financeiros com a menor média de 3,20, cuidados de saúde e sociais: disponibilidade e qualidade com 3,17 e por fim, transporte apresentou uma média de 3,12.

Figura 5 - Valores obtidos nas facetas do domínio meio ambiente através do instrumento WHOQOLbref dos idosos inseridos na Universidade Aberta à Terceira Idade, $n=132$. 2016-2017.

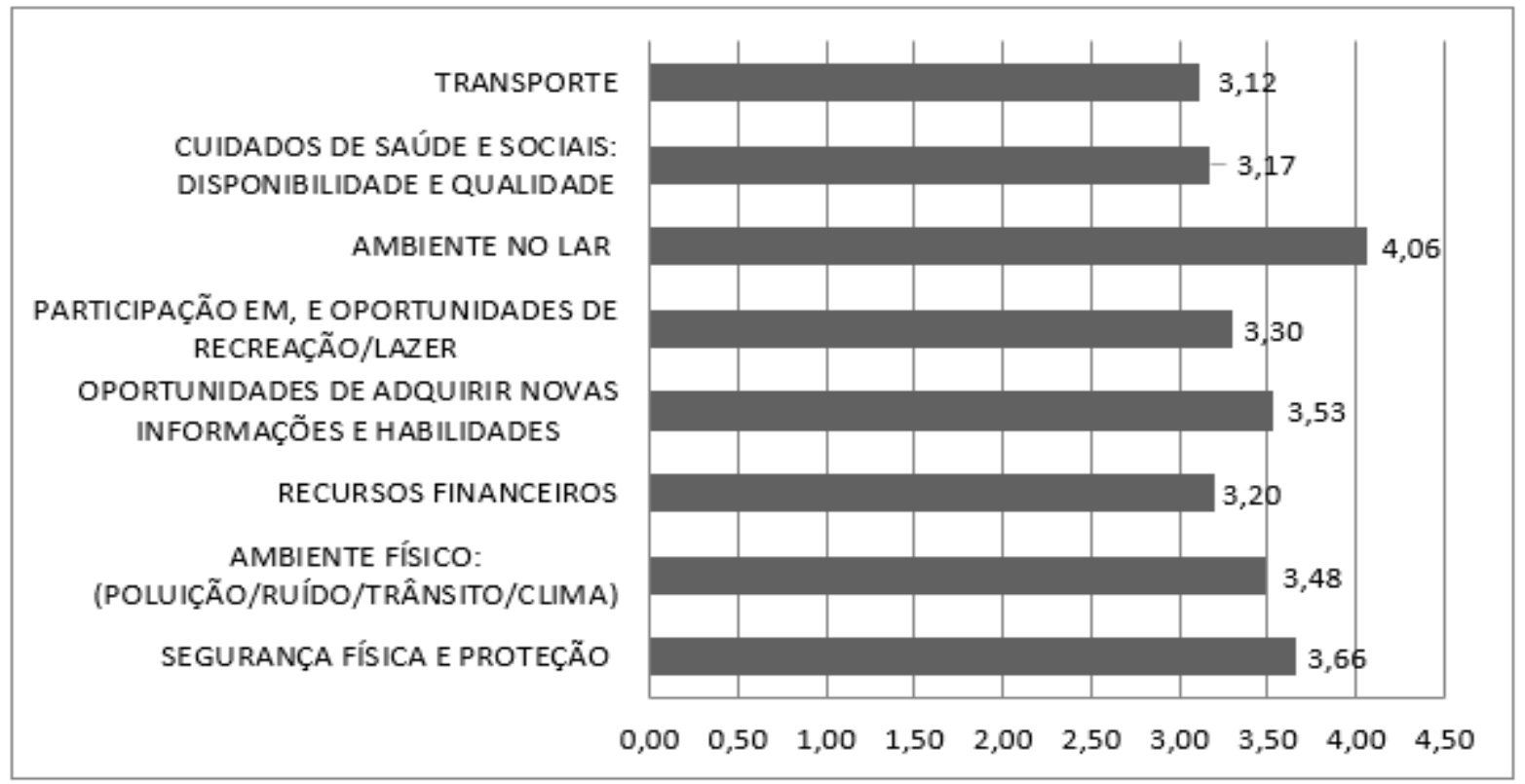

Fonte: Silva MB, et al., 2020.

Na avaliação da QV através do módulo WHOQOL-old, conforme apresentado na Tabela 1, as facetas que apresentaram maiores escores para QV foram habilidades sensoriais, com uma média de 4,03 e participação social com 3,82. A faceta morte e morrer teve 3,61 e autonomia ficou com 3,58. Intimidade apresentou uma média de 3,73, assim como atividades passadas, presentes e futuras também ficou com média de 3,73 . 0 escore de média total apresentado pelas facetas foi de 3,75 . 
Tabela 1 - Valores obtidos nas facetas através do módulo WHOQOL-old dos idosos inseridos na Universidade Aberta à Terceira Idade, $n=132$. 2016-2017.

\begin{tabular}{ccc}
\hline Facetas & Média & Porcentagem \\
\hline Habilidades Sensoriais & 4,03 & $76 \%$ \\
Autonomia & 3,58 & $65 \%$ \\
Atividades passadas, presentes e futuras & 3,73 & $68 \%$ \\
Domínio participação social & 3,82 & $71 \%$ \\
Morte e morrer & 3,61 & $65 \%$ \\
Intimidade & 3,73 & $68 \%$ \\
Qualidade de vida geral & 3,75 & $69 \%$ \\
\hline Total & 3,75 & - \\
\hline
\end{tabular}

Fonte: Silva MB, et al., 2020.

\section{DISCUSSÃO}

O presente estudo apresentou predominâncias de características específicas da população bastante similares ao estudo de Roque FP, et al. (2011) realizado com 460 idosos participantes de uma Universidade Aberta à Terceira Idade, igualmente localizada no Estado de Alagoas, que também reportou a predominância do sexo feminino $(93,3 \%)$ e idade entre 60 e 69 anos $(41,57 \%)$. Além de o processo do envelhecimento retratar domínio feminino pela expectativa de vida da mulher ser mais alta que do homem, pode também se esclarecer pela falta de interesse da população masculina em relação às atividades lúdicas, educacionais e sociais, onde o contexto cultural refere que a participação desse tipo de atividade é caracterizada como feminina.

Assim como os estudos de Marchiore FG, et al. (2013) e Reis SP, et al. (2015), de acordo com os resultados obtidos na (Figura 1), nota-se um maior escore para a percepção da qualidade de vida em relação à satisfação com a saúde, que se apresentou regular, divergindo com os dados trazidos pelo IBGE (2010), onde $45,5 \%$ dos idosos declararam-se satisfeitos com seu estado de saúde. Para Dawalibi NW, et al. (2014) era esperado que eles tivessem uma melhor percepção de sua qualidade de vida, visto que são idosos que frequentam Universidades Abertas à Terceira Idade, o que os particularizam dos demais idosos da população em geral, no sentido de busca por conhecimento e cuidados com a saúde.

$\mathrm{Na}$ avaliação dos domínios do instrumento WHOQOL-bref, todos os escores se apresentaram com uma média regular, o maior escore foi o do domínio psicológico, que sofreu influência da faceta autoestima, que foi conceituada com a maior pontuação dentre todas as facetas do estudo em questão.

Para Ordonez TN e Cachioni M (2011) e outros estudiosos como Silva RAG (2013) que verificou a presença da depressão e da autoestima em idosos participantes de uma Universidade Aberta à Maturidade (UAMA) no Estado da Paraíba, encontrou resultado similar a este estudo, com aumento da autoestima dos idosos entrevistados, visto que as atividades oferecidas nas UAMA possibilitam formação de novos ciclos de amizade, diminui os estresses e as doenças crônicas, como a depressão ao longo do envelhecimento.

O domínio meio ambiente teve o menor escore, transporte destacou-se com a menor pontuação em todas as facetas deste estudo. No entanto, a pesquisa de Silva SLP (2012), realizada no Estado da Paraíba, descreve que a maioria dos idosos entrevistados se mostrou satisfeita ou até muito satisfeita com o transporte, divergindo deste estudo. $\mathrm{O}$ que pode ser justificado pelas divergências sociais e, por se tratar de um Estado com índice de desenvolvimento humano (IDH) mais elevado que o do Estado de Alagoas.

A faceta ambiente no lar foi a única do domínio meio ambiente que obteve um escore satisfatório. A família é considerada por Witter C e Camilo AR (2011) uma rede social importante para promover e manter atividades de convívio social, pois, proporciona a relação interpessoal entre os familiares, outras pessoas e o idoso, o que corrobora com o envelhecimento saudável.

No domínio físico, a faceta dependência de medicamentos ou tratamento colaborou para um escore abaixo do satisfatório. Segundo Costa SC e Pedroso ERP (2011) com o processo do envelhecimento, há um aumento de doenças crônicas, o que favorece contínuo consumo de medicamentos entre os idosos. 
Além disso, os fármacos é a principal forma de intervenção usada no modelo de saúde atual. Com isso, há um consumo excessivo, onde medidas precisam ser avaliadas quanto ao seu risco/benefício, pois o uso desmoderado pode influenciar significativamente na QV dos idosos (COSTA SC e PEDROSO ERP, 2011).

O domínio relações sociais teve um escore bem próximo do satisfatório, porém, teve grande influência negativa da faceta atividade sexual, que foi a única faceta deste domínio que se apresentou insatisfatória. Para Alencar DL, et al. (2014) há diversos fatores que influenciam a sexualidade do idoso, como: a ausência do parceiro devido à viuvez, ocorrência de doença, mudanças na fisiologia sexual e o uso de medicamentos. Com isso, é justificada a insatisfação do idoso com sua sexualidade.

Neste mesmo domínio, as relações pessoais encontram-se acima da média, mostrando-se satisfatória. Em um estudo que avaliou a percepção dos idosos sobre a qualidade de vida de Paskulin LMG, et al. (2010), as relações pessoais foram citadas como convívio com os amigos e familiares. Sentir-se útil perante a sociedade foi um fator associado a esse tipo de relação. Isso corrobora para o elevado escore, considerando que os idosos em questão são ativos e estão inseridos socialmente através da Universidade Aberta à Terceira ldade.

Os resultados obtidos no questionário WHOQOL-old evidenciaram as facetas morte e morrer e autonomia com os menores índices: 3,61 e 3,58, respectivamente. Inquietações, preocupações e temores sobre o processo morte e morrer foram observados na maioria dos entrevistados. Segundo Reis SP, et al. (2015) este fato pode estar ligado à dificuldade de acesso aos serviços de saúde.

Um estudo realizado por Paula GR, et al. (2017) obteve um resultado equivalente, onde a faceta morte e morrer teve um escore insatisfatório. Para Araújo LP, et al. (2009) isso é efeito do número de entrevistados ser composto em sua maioria por idosos mais jovens. Mesmo tendo consciência que a morte é inevitável, os entrevistados assumem seus medos do desconhecido e das condições indignas que podem acompanhar o processo de morrer. Apesar da jovialidade dos idosos em questão, a faceta autonomia não obteve um escore satisfatório. As limitações físicas, psicoemocionais e baixa escolaridade são fatores citados por Bento JA e Lebrão ML (2014) que afetam significativamente a autonomia pessoal.

Os aspectos bioéticos envolvidos na autonomia do idoso foram descritos por Saquetto M, et al. (2013) e concluiu que tem sido uma temática bastante discutida nos campos da geriatria e gerontologia. Pois, com o aumento da expectativa de vida, torna-se imprescindível que medidas que fomentam o envelhecimento ativo sejam elaboradas, voltando-se cada vez mais à valorização da autonomia como função essencial à saúde da pessoa idosa.

\section{CONCLUSÃO}

Conclui-se que apesar de serem considerados idosos ativos e se perceberem com boa da qualidade de vida, foi evidenciada uma média regular em grande maioria dos domínios e facetas e, que as questões em relação ao meio ambiente como transporte, recursos financeiros, poluição, trânsito, clima, oportunidades de lazer e segurança implicaram negativamente, o que ressalta a importância dos fatores externos na QV da sociedade em geral. A autoestima preservada mostra que envelhecer não é sinônimo de não aceitação dos declínios naturais da longevidade. Diante disso, ressalto a importância de novos estudos para aprimorar conhecimentos acerca da saúde e da qualidade de vida da pessoa idosa.

\section{LIMITAÇÃO DO ESTUDO}

Este estudo apresenta uma limitação referente a definição da amostra por ser considerada um fator limitante, tendo em vista o fato de não ser aleatória e por ter sido considerada apenas a população idosa mais ativa.

\section{REFERÊNCIAS}

1. ALENCAR DL, et al. Fatores que interferem na sexualidade de idosos: uma revisão integrativa. Revista Ciência \& Saúde Coletiva, 2014; 19(8): 3533-3542.

2. ARAÚJO LP, et al. Medo à morte e ao morrer em idosas institucionalizadas e não institucionalizadas. Acta Scientiarum. Human and Social Sciences, 2009; 31(2): 213-218. 
3. BENTO JA, LEBRÃO ML. Sufficiency of income perceived by the elderly in the city of São Paulo/Brazil. Ciênc Saúde Coletiva, 2014; 18(8): 2229-38.

4. COSTA SC, PEDROSO ERP. A prescrição de medicamentos para idosos internados em serviço de clínica médica: atualização. Rev Med Minas Gerais, 2011; 21(2): 201-1.

5. DAWALIBI NW, et al. Índice de desenvolvimento humano e qualidade de vida de idosos frequentadores de universidades abertas para a terceira idade. Psicol. Soc, 2014; 26(2): 496-505.

6. IBGE - Instituto Brasileiro de Geografia e Estatística. Síntese dos Indicadores Sociais: uma análise das condições de vida da população brasileira. Censo demográfico 2010. Brasília 2010.

7. LINDER JUNIOR E, TRINDADE JLA. Avaliação da qualidade de vida de idosos em um município do Sul do Brasil. Rev. bras. geriatr. Gerontol, 2013; 16(3): 473-479.

8. MARCHIORI FG, et al. Qualidade de vida entre idosos com e sem companheiro. Rev enfer UFPE online. Recife, 2013; 7(4): 1098-106.

9. MINISTÉRIO DA SAÚDE. Vigilância de Doenças Crônicas Não Transmissíveis. Plano de Ações Estratégicas para o Enfrentamento das Doenças Crônicas Não Transmissíveis (DCNT) no Brasil 2011-2022. Brasília: Ministério da Saúde; 2011. 160p.

10. MIRANDA GMD, et al. O envelhecimento populacional brasileiro: desafios e consequências sociais atuais e futuras. Rev. bras. geriatr. Gerontol, 2016; 19(3): 507-519.

11. ORDONEZ TN e CACHIONI M. Motivos para frequentar um programa de educação permanente: relato dos alunos da universidade aberta à terceira idade da Escola de Artes, Ciências e Humanidades da Universidade de São Paulo. Rev. bras. geriatr. Gerontol, 2011; 14(3): 461-474.

12. PASKULIN LMG, et al. Percepção de pessoas idosas sobre qualidade de vida. Acta Paul Enferm, 2010; 23(1): 1017.

13. PAULA GR, et al. Qualidade de vida para avaliação de grupos de promoção da saúde. Rev. Bras. Enferm, 2016; 69(2): 242-249.

14. REIS SP, et al. Estudo da qualidade de vida de idosos não institucionalizados. JCBS, 2015; 1(2): 56-60

15. ROQUE FP, et al. Perfil socioeconômico-cultural de uma universidade aberta à terceira idade: reflexo da realidade brasileira? Rev. bras. geriatr. gerontol. 2011; 14(1): 97-108.

16. SAQUETTO M, et al. Aspectos bioéticos da autonomia do idoso. Rev. Bioét, 2013; 21(3): 518-524.

17. SOUZA e SOUZA M. Desafios do envelhecimento populacional: como as legislações destinadas aos idosos têm lidado com essa nova demanda? Estudos Interdisciplinares sobre o Envelhecimento, 2015; 20(1): 159-175.

18. SILVA RAG. Depressão e autoestima em idosos participantes da Universidade Aberta a Maturidade: Um estudo de prevalência. Trabalho de Conclusão de Curso (Graduação em Fisioterapia) - Universidade Estadual da Paraíba, Campina Grande, 2013. 29p.

19. SILVA SLP. Avaliação da qualidade dos serviços de transporte interestadual: uma análise na percepção dos idosos. Trabalho de Conclusão de Curso (Bacharelado em Administração) - Universidade Federal da Paraíba, João Pessoa, 2012. 73p.

20. TAVARES DMS, DIAS FA. Capacidade funcional, morbidades e qualidade de vida de idosos. Texto contexto enferm, 2012; 21(1): 112-20

21. TIER GC, et al. Condições de saúde dos idosos na Atenção Primária a Saúde. Rev Rene, 2014; 15(4): 668-75.

22. WHOQOL GROUP. The world health organization quality of life assessment (WHOQOL): position paper from the world health organization. Soc Sci Med, 1995; 41(10): 1403-1409.

23. WITTER C, CAMILO AR. Família e envelhecimento. In WITTER, C; BURITI, M.A \& WITTER, GP. Envelhecimento e contingências da vida. Campinas-SP: Alínea, 2011:83. 102p. 